\title{
Analysis of 3D formation pressure based on logging data
}

\author{
Ya-Nan Sheng ${ }^{1}\left(\right.$ i) $\cdot$ Zhi-Chuan Guan $^{1} \cdot$ Kai Wei $^{2}$
}

Received: 4 March 2016/ Accepted: 18 June 2016/Published online: 2 July 2016

(C) The Author(s) 2016. This article is published with open access at Springerlink.com

\begin{abstract}
A method for constructing regional 3D formation pressure based on logging data was established in this paper. It aims to deal with the problem of constructing regional 3D formation pressure in the block which is short of the seismic data or has poor quality of seismic data. To begin with, logging data and core data of drilled wells were collected. Making full use of the fine continuity and accurate resolving capability of logging data, we calculated the formation pressure of drilled wells based on the Eaton law. In addition, based on the concept of digital strata, the concept of formation matrix was put forward. What is more, formation matrix of the aimed layer group was built by making use of the drilled well formation pressure based on the epitaxial transfer algorithm. Finally, we realized the description of the regional 3D formation pressure. To verify the reliability of the method, one drilled well formation pressure was derived from the 3D formation pressure. It was compared with the pressure interpreted by the logging data. The maximum error was less than $5 \%$. Finally, by using the computer visible language, the software of describing 3D formation pressure was developed. And it is conductive to drilling engineer to predict forma-
\end{abstract}

Ya-Nan Sheng

shengyanan_upc@163.com

1 School of Petroleum Engineering, China University of Petroleum (East China), Qingdao 266580, China

2 School of Petroleum Engineering, Yangtze University, Wuhan 434023, China tion pressure which can provide the basis for the casing design and the selection of the drilling fluid density.

Keywords 3D formation pressure · Formation matrix · Epitaxial transfer algorithm $\cdot 3 \mathrm{D}$ visualization software

\section{Introduction}

Results of geological study on the Niudong block of Qinghai Oilfield indicated that there were many folds and fault zones in the block, which made it difficult to predict the formation pressure. The vague understanding of the formation pressure caused many complex situations or drilling risks. The conventional simple one-dimensional pressure prediction result had been very difficult to meet the needs of drilling engineering. Analysis of 3D regional formation pressure should be studied in order to improve the prediction accuracy of the formation pressure. There have been many 3D formation pressure prediction methods which include logging-constrained seismic inversion method, one-point hypothesis and spatial interpolation algorithm (Xueguo 2005; Honghai et al. 2011; Zhi et al. 2013). However, these methods are all based on the seismic data. If the block is lack of seismic data or the quality of seismic data is poor, the calculation accuracy of 3D formation pressure based on seismic data is difficult to guarantee. With the development of block, we can obtain more and more data of the drilled wells such as logging, core data and well history information. However, the method for constructing the 3D formation pressure based on logging data has yet been put forward. In this paper, a new method was proposed. The spatial distribution of 3D formation pressure can be more clearly understood by this method. 


\section{The algorithm of 3D formation pressure}

\section{Theoretical basis of the algorithm}

\section{Formation pressure calculation model}

The prediction methods of formation pressure were summarized and classified. These methods can be classified into three categories according to the order of drilling time: pressure prediction before drilling, pressure monitoring while drilling and pressure detection after drilling. They can also be divided into three categories based on different sources of data: the pressure prediction based on seismic data, the pressure monitoring by PWD and pressure detection by using the logging data. Since then, many pressure calculation models had been established, such as Eaton law (Eaton 1972), Dutta law (Dutta 2002) and Fillippone law (Fillippone 1979). The pressure calculation model should be chosen to fit the characteristics of geology in the block.

The formation pressure of drilled wells was calculated by different prediction models. Then, the calculated results and measured pressures were compared. Finally, we found that the simple Eaton method was suitable for predicting formation pressure in this block.

\section{The concept of formation matrix}

The digital formation is a comprehensive research system which is gradually developed with the research of the 3D digital GIS (Zhang et al. 1997), geological modeling (Wu et al. 2005) and 3D visualization in drilling operations (Victor 1997). In order to realize the scientific management and application of the digital formation, this paper put forward the concept of the formation matrix.

The formation matrix consists of the space position matrix and the geological attribute parameter matrix (as shown in Fig. 1). Under the Cartesian coordinates, the formation matrix consists of four matrices: $X, Y, Z$ and $P$ which, respectively, represent $x$ position matrix, $y$ position matrix, $\mathrm{z}$ position matrix and attribute parameter matrix. The description of 3D formation pressure was transformed into the construction of formation matrix.

\section{Realization of algorithm}

\section{The deployment of virtual wells}

The purpose of marking stratigraphic grid is to arrange the virtual wells in the block. Based on the well pattern in the plane, the $\mathrm{X}$ and $\mathrm{Y}$ directions are divided into grid number of $N_{X}$ and $N_{Y}$ which, respectively, indicate the density of virtual well pattern in the two directions. The $\mathrm{Z}$ directions are divided into grid number of $N_{H}$ which represents the discrete point density of formation pressure along the vertical well depth. The density of virtual wells increases with the increase in the mesh numbers. And the matrix of the formation pressure increases with the increase in the density of virtual wells. The greater the formation matrix, the accuracy of 3D formation pressure is higher. Then, the $\left(N_{X} \times N_{Y} \times N_{H}\right)$ matrix is used to represent the spatial distribution of formation. The matrix $P(i, j, k)$ represents the pressure of a rectangular region in the formation. The pressure in the center point of the rectangle is used to represent the pressure of the rectangular region. The grid division of the target strata is shown in Fig. 2.

\section{Formation pressure calculation of virtual well}

Theory and practice have proved that (Catuneanu 2009) in the same geological stratum under the same deposition conditions, it should produce the same interpretation results of lithology, seismic or logging. So the formation pressure of the layer group in the block is correlated. In the paper by Zhichuan and Kai 2013, based on the drilled well logging data, the epitaxial transfer algorithm was established to calculate the target well formation pressure in the same layer group.

Assuming that this block has been drilled $N$ wells, the location coordinates of the $x$-th drilled well are $\left(X_{x}, Y_{x}\right)$, $x=1,2, \ldots, N$. There are $m$ discrete points of formation pressure of the $x$-th drilled well in the $i$-th layer group. The corresponding formation matrix is:

$$
P_{x}^{i}=\left[\begin{array}{cc}
H_{x 1}^{i} & p_{x 1}^{i} \\
\vdots & \vdots \\
H_{x j}^{i} & p_{x j}^{i} \\
\vdots & \vdots \\
H_{x m}^{i} & p_{x m}^{i}
\end{array}\right]
$$

If the location coordinates of the target of well are $\left(X_{O}, Y_{O}\right)$, according to epitaxial transfer algorithm, the formation pressure of the $x$-th drilled well in the $i$-th layer group was transplanted to the target well:

$\overline{P_{x}^{i}}=\left[\left[\left(H_{O u}^{i}-\frac{H_{x u}^{i}\left(H_{x d}^{i}-H_{x u}^{i}\right)}{H_{x d}^{i}-H_{x u}^{i}}\right)\left[\begin{array}{c}1 \\ \vdots \\ 1 \\ \vdots \\ 1\end{array}\right]_{m \times 1}+\frac{H_{O d}^{i}-H_{O u}^{i}}{H_{x d}^{i}-H_{x u}^{i}}\left[\begin{array}{c}H_{x 1}^{i} \\ \vdots \\ H_{x j}^{i} \\ \vdots \\ H_{x m}^{i}\end{array}\right]\right] \frac{1}{d_{x}^{t}}\left[\begin{array}{c}p_{x 1}^{i} \\ \vdots \\ p_{x j}^{i} \\ \vdots \\ p_{x m}^{i}\end{array}\right]\right]$

where $\mathrm{dx}=\sqrt{\left(\mathrm{X}_{0}-\mathrm{X}_{x}\right)^{2}+\left(\mathrm{Y}_{o}-\mathrm{Y}_{x}\right)^{2}}$ is the horizontal distance from point $\left(\mathrm{X}_{0}, \mathrm{Y}_{0}\right)$ to point $(\mathrm{X} x, \mathrm{Y} x), x=1,2, \ldots$, 
Fig. 1 Formation matrix

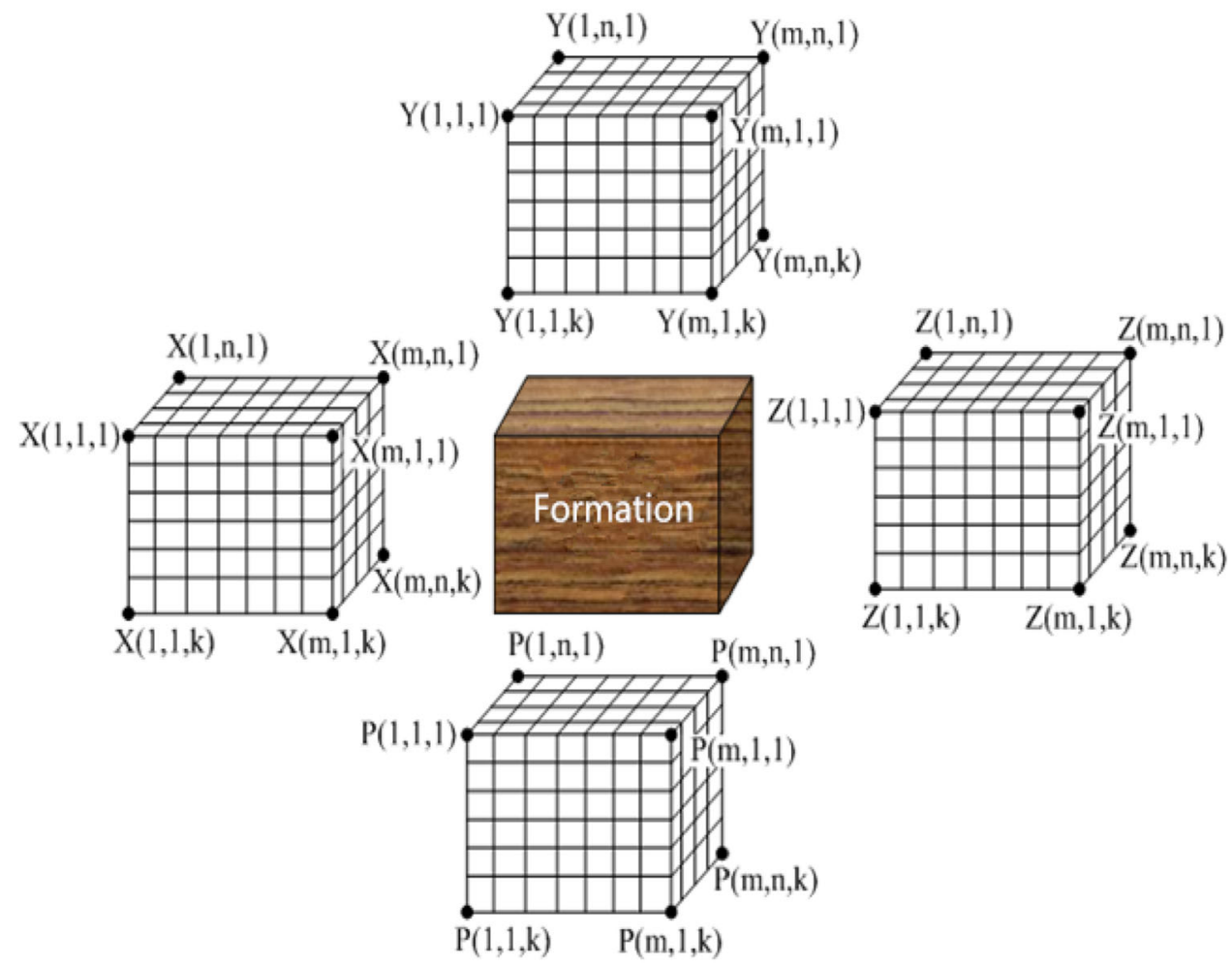

$\mathrm{N}$. The $t$ which is called the weighted exponent is a constant greater than 0 .

All of the drilled well formation pressures in the $i$-th layer group were transplanted to the target well. Finally, we got the formation pressure matrix of the target well: and pressure matrixes are three-dimensional matrixes, and the matrix size is $\mathrm{N}_{\mathrm{x}} \times \mathrm{N}_{\mathrm{Y}} \times \mathrm{N}_{\mathrm{H}}$. The first and second columns in $\left(\mathrm{N}_{\mathrm{X}} \times \mathrm{N}_{\mathrm{Y}} \times \mathrm{N}_{\mathrm{H}}\right)$ represent location coordinates of the virtual wells, and the third column represents the interpolation point number along the vertical well depth. The

$\left.\overline{P_{x}^{i}}=\left[\left(H_{O u}^{i}-\frac{H_{x u}^{i}\left(H_{x d}^{i}-H_{x u}^{i}\right)}{H_{x d}^{i}-H_{x u}^{i}}\right)\left[\begin{array}{c}1 \\ \vdots \\ 1 \\ \vdots \\ 1\end{array}\right]_{m \times 1}+\frac{H_{O d}^{i}-H_{O u}^{i}}{H_{x d}^{i}-H_{x u}^{i}}\left[\begin{array}{c}H_{x 1}^{i} \\ \vdots \\ H_{x j}^{i} \\ \vdots \\ H_{x m}^{i}\end{array}\right]\right] \frac{\frac{1}{d_{x}^{t}}}{\sum_{x}^{N} \frac{1}{d_{x}^{t}}}\left[\begin{array}{c}\sum_{x}^{N} p_{x 1}^{i} \\ \vdots \\ \sum_{x}^{N} p_{x j}^{i} \\ \vdots \\ \sum_{x}^{N} p_{x m}^{i}\end{array}\right]\right]$

\section{Construction of formation matrix}

According to the method established above, the formation matrix of all of the virtual wells in the block can be set up. The space position matrix $\mathrm{H} 3\left(\mathrm{~N}_{\mathrm{x}} \times \mathrm{N}_{\mathrm{Y}} \times \mathrm{N}_{\mathrm{H}}\right)$ is formed by combining the depth matrix $H$ with the matrix $X$ and matrix $Y$. By the same method, the pressure matrixes $P$ of the virtual wells are reconstructed, and the formation pressure matrixes P3 $\left(\mathrm{N}_{\mathrm{X}} \times \mathrm{N}_{\mathrm{Y}} \times \mathrm{N}_{\mathrm{H}}\right)$ are formed. Both the depth matrixes pressure value $\mathrm{P} 3(\mathrm{i}, \mathrm{j}, \mathrm{k})$ indicates the formation pressure value at the space position of $\mathrm{H} 3(\mathrm{i}, \mathrm{j}, \mathrm{k})$. Then, the formation matrix of the target layer group can be obtained, which can realize the description of $3 \mathrm{D}$ formation pressure.

\section{Visualization software of $3 D$ formation pressure}

Based on the above algorithm and computer visible language (Xiong 2007; Zhichuan and Kai 2013; Sanstrom and 


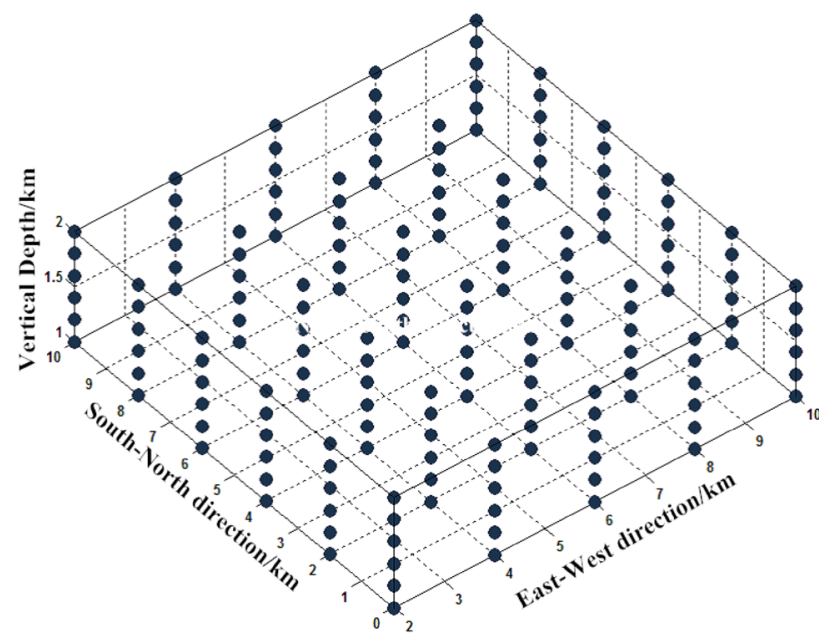

Fig. 2 Gridding the target stratum

Hawkins 2000), the visualization software of building the 3D formation pressure was programed by MATLAB. We can obtain four results through the software: (1) 3D formation pressure of the target layer group in the block, (2) two-dimensional transverse distribution of formation pressure at a depth, (3) two-dimensional longitudinal formation pressure profile of the well and (4) formation pressure profile along the trajectory of directional well.

\section{Example}

In order to verify the reliability of the method and the software, this paper regarded the Niudong block of Qinghai Oilfield as the target area. There have been five drilled wells in the block. Firstly, the logging data and core data of the five drilled wells were collected. We chose N1 as the target well and the rest four wells N2, N3, N4, N5 as the drilled adjacent wells. The well deployment diagram is shown in Fig. 3. The ups and downs of the target layer group are shown in Table 1.

Steps of constructing regional 3D formation pressure are divided into the following five parts:

(1) The formation pressure of the four drilled wells were calculated based on the Eaton law. The results are shown in Fig. 4.

(2) The deployment of virtual wells.

(3) Formation pressure calculation of virtual well.

The target well formation pressure can be gotten based on the method established above (Fig. 5. The maximum error between the transplantation pressure with the logging interpretation pressure was $4.2 \%$, as shown in Fig. 6. This showed that the algorithm proposed in this paper can meet the requirements of drilling engineering. In the same way,

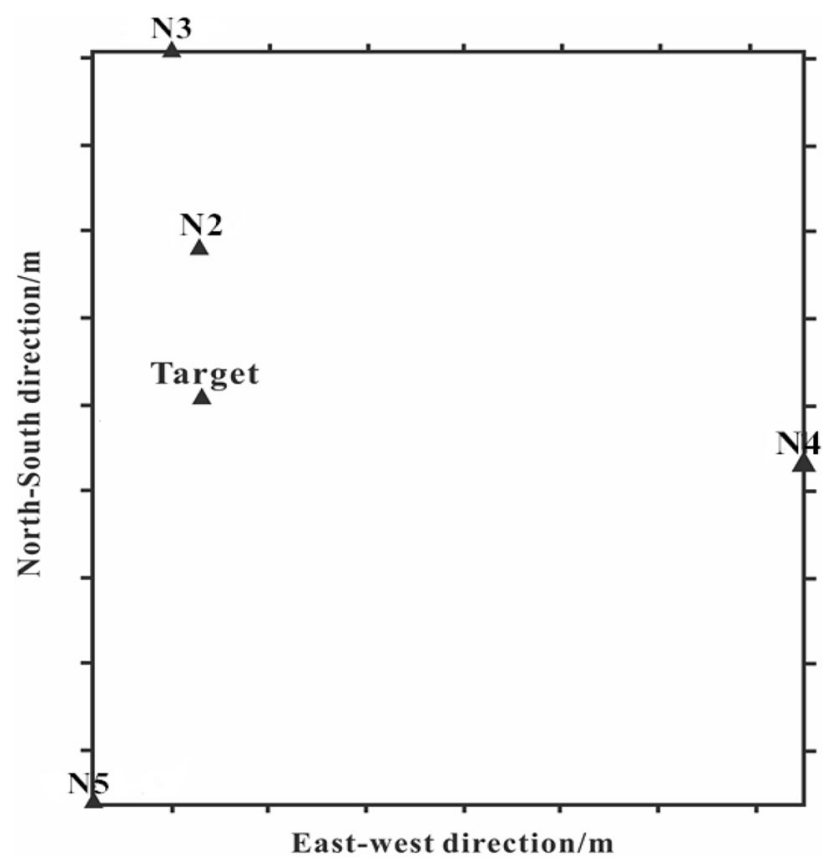

Fig. 3 Adjacent wells coordinate

Table 1 The bound of the target stratum

\begin{tabular}{lll}
\hline Well & Top depth/m & Bottom depth/m \\
\hline N2 & 1620 & 2160 \\
N3 & 1600 & 2090 \\
N4 & 1424 & 1928 \\
N5 & 1340 & 1630 \\
N1 & 1524 & 2050 \\
\hline
\end{tabular}

we can get the formation pressure of any one of the virtual wells in the block.

(4) Visualization of 3D formation pressure.

According to the software developed in this paper, the $3 \mathrm{D}$ formation pressure of one certain layer group was obtained. And the pressure profile along with the well depth of the target well was extracted from the 3D formation pressure. The results are shown in Figs. 7 and 8.

(5) Algorithm verification.

In order to verify the accuracy of the calculation, the formation pressure profile of the target well was extracted from the 3D formation pressure. Then, we compared it with the result of logging interpretation pressure. The results are shown in Table 2. The maximum error was less than $5 \%$. The accuracy of this method can meet the engineering requirement. We can obtain accurate space distribution of 
Fig. 4 Pore pressure profiles of four drilled wells

Fig. 5 Virtual well deployment diagram

Fig. 6 Comparison of transplantation pressure and logging interpretation pressure
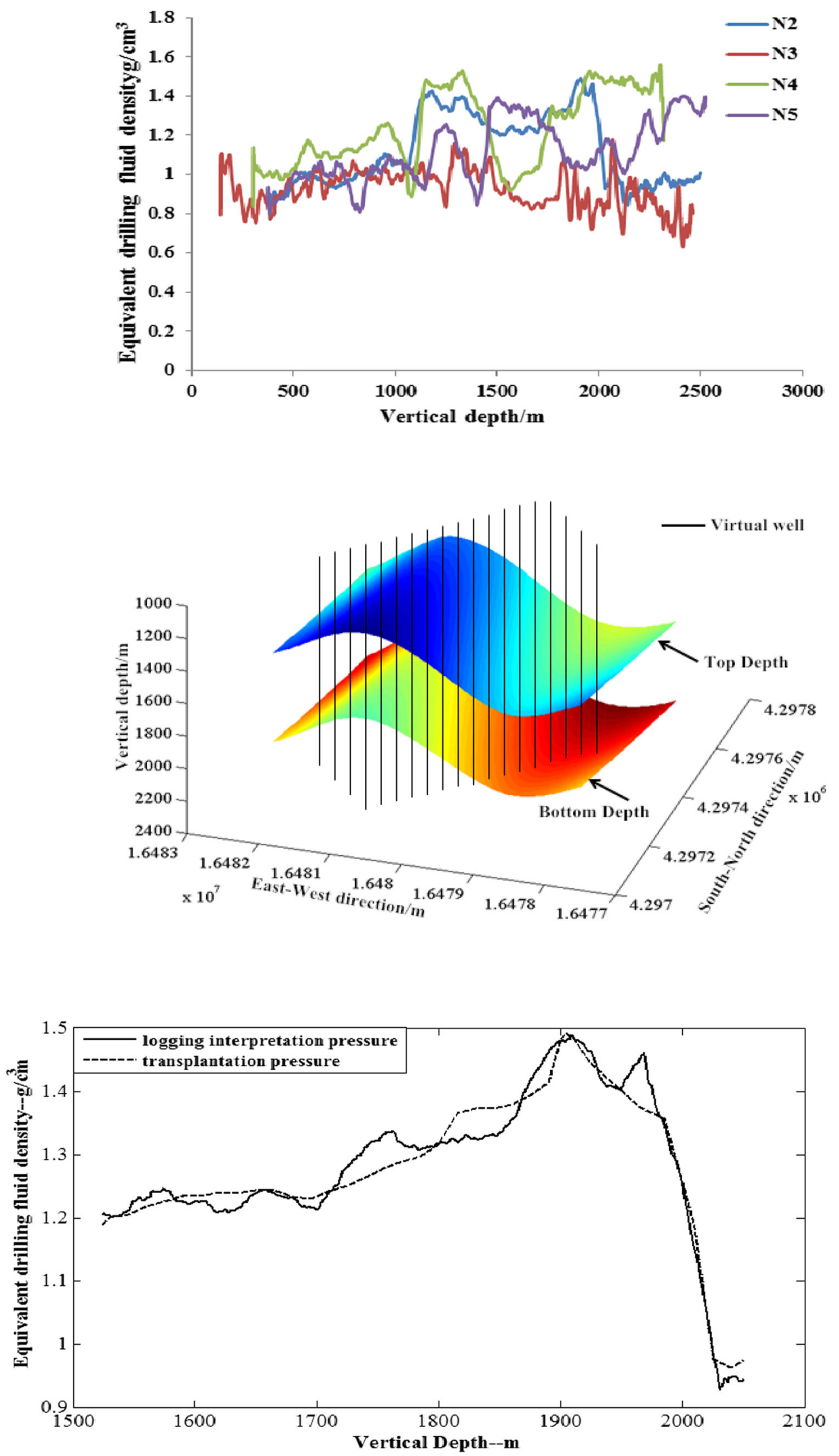
Fig. 7 3D formation pressure of the target layer group
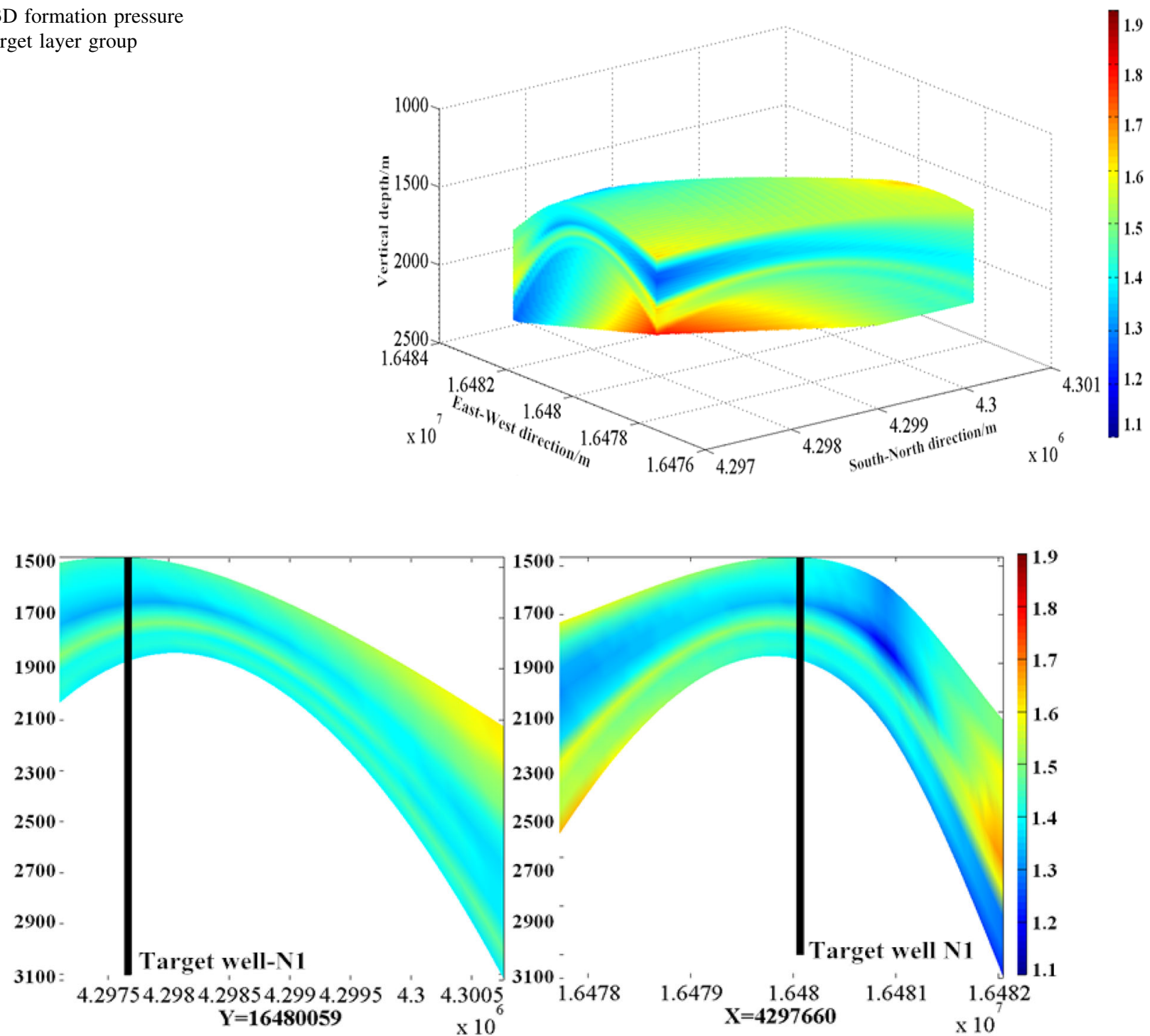

Fig. 8 Formation pressure profile of target well N1

Table 2 Verification of the algorithm

\begin{tabular}{llll}
\hline $\begin{array}{l}\text { Depth } \\
(\mathrm{m})\end{array}$ & $\begin{array}{l}\text { Transplantation } \\
\text { pressure }\end{array}$ & $\begin{array}{l}\text { Logging interpretation } \\
\text { pressure }\end{array}$ & $\begin{array}{l}\text { Error } \\
(\%)\end{array}$ \\
\hline 1550 & 1.22 & 1.24 & 1.6 \\
1700 & 1.24 & 1.19 & 4.2 \\
1800 & 1.30 & 1.26 & 3.2 \\
1900 & 1.51 & 1.49 & 1.3 \\
2000 & 1.15 & 1.2 & 4.1 \\
\hline
\end{tabular}

regional formation pressure by this method. This is conductive to drilling engineer to predict pore pressure which can provide the basis for the casing design and the selection of the drilling fluid density.

\section{Conclusions}

(1) The concept of formation matrix was put forward. The description of 3D formation pressure was transformed into the construction of formation matrix. A method for constructing regional formation pressure based on the drilled wells logging data was established. The 3D formation pressure can be constructed layer by layer by using this method.

(2) The visualization software of 3D formation pressure was programed. By this software, the regional formation pressure can be displayed layer by layer, rotated or displayed in arbitrary cutting section, etc. This is conductive to drilling engineer to predict pore pressure which can provide the basis for the casing design and the selection of the drilling fluid density. 
Acknowledgments The authors would like to acknowledge the academic and technical support of China University of Petroleum (East China). This paper is supported by the National Natural Science Foundation of China (No. 51574275), the Graduate Innovation Project Foundation (CX2013011) and CNOOC Research Institute "Research on Key Technologies of horizontal well drilling and well completion in high temperature and high pressure and high sulfur gas field in overseas BD"(YXKY-2015-ZY-12).

Open Access This article is distributed under the terms of the Creative Commons Attribution 4.0 International License (http:// creativecommons.org/licenses/by/4.0/), which permits unrestricted use, distribution, and reproduction in any medium, provided you give appropriate credit to the original author(s) and the source, provide a link to the Creative Commons license, and indicate if changes were made.

\section{References}

Catuneanu O (2009) Principles of sequence stratigraphy [M]. WuYinye translated. Petroleum Industry Press, Beijing

Dutta NC (2002) Deepwater geohazard perdiction using prestack in version pf large offset $\mathrm{P}$ wave data and rock model [J]. Lead Edge Geophys 21(2):193-198

Eaton BA (1972) Graphical method predicts geopressures worldwide. World Oil 182:51-56
Fillippone WR (1979) On the prediction of abnormally pressured sedimentary rocks from seismic data. J Petrol Techn

Honghai F, Zhi Y, Rongyi J et al (2011) Investigation on threedimensional overburden pressure calculation method. Chin $\mathbf{J}$ Rock Mech Eng 30(2):3878-3883

Sanstrom WC, Hawkins MJ (2000) Perceiving drilling learning through visualization. IADC/SPE 62759

Victor J (1997) Taking advantage of 3-dimensional visualization in drilling operations. IADC/SPE 37591

Wu Q, Xu H, Zou X (2005) An effective method for 3D geological modeling with multi-source data integration [J]. Comput Geosci 31(1):35-43

Xiong Z (2007) Study on the technology of 3D engineering geological modeling and visualization. Wuhan: The Chinese Academy of Sciences (Institute of Rock\&Soil Mechanics)

Xueguo C (2005) Technology and applications of 3D formation pressure prediction. Petroleum Drill Tech 33(3):13-15

Zhang J, Liu J, Li D et al (1997) Technical approach to realistic terrain generation [J]. J Image Graphics 8(9):638-645

Zhi Y, Honghai F, Zhang G (2013) Calculation method and distribution of three-dimensional pore pressure in changshaling structural belt. Oil Drill Prod Technol 1:51-55

Zhichuan G, Kai W (2013a) A new method for establishing regional formation pressure profile based on drilled wells data $[\mathrm{J}]$. J China University Petroleum 05:71-75

Zhichuan G, Kai W (2013b) Establishing the formation pressure profile of predrill well based on adjacent wells data $[\mathrm{J}]$. J China University Petroleum 37(5):71-75 